\title{
Pflegereform nicht isoliert betrachten - Die Probleme reichen weit über die Pflegeversicherung hinaus
}

ROBERT PAOUET

Dr. Robert Paquet ist Freier Journalist und Berater, Berlin

Das "Jahr der Pflege" endete für viele enttäuschend. Wenn die Erwartungen allerdings nicht so hoch geschraubt worden wären (z. B. nach den vollmundigen Formulierungen des Koalitionsvertrages und Minister Röslers "Pflegedialog" etc.) hätte man das jetzt erreichte Ergebnis gar nicht so schlecht gefunden. Etwas für die Demenzkranken zu tun, geht in die richtige Richtung. Und gegen die Förderung der Eigenvorsorge kann man ebenfalls wenig einwenden. Dass das für Leistungsverbesserungen zur Verfügung stehende Finanzvolumen kleiner ausgefallen ist, als viele erwartet hatten, kann angesichts der Selbstblockade der Koalition bei diesem Thema kaum verwundern. - Dabei konzentrierte sich die Diskussion in den letzten zwei Jahren vor allem auf Fragen der Finanzierung der Pflegeversicherung, auf die Bewältigung des Fachkräftemangels und auf die Umsetzung des "neuen« Pflegebegriffs. Der aktuelle Stand der Debatte ist daher Anlass für den Hinweis auf weitere Aspekte der Pflegepolitik, die bisher zu wenig berücksichtigt worden sind'.

\section{Pflege und Alterssicherung}

Nach dem ursprünglichen Konzept sollte die Pflegeversicherung zur Finanzierung der zusätzlichen Kosten beitragen, die auf die Versicherten durch die Pflegebedürftigkeit zukommen. Realisten haben dabei nie geglaubt, dass die Sätze der Pflegeversicherung reichen könnten, alle pflegebedingten Kosten zu decken. Auch die Forderung nach einer
Vollfinanzierung etwa der stationären Pflege war immer unrealistisch. Es wurde stets unterstellt, dass der Pflegebedürftige aus eigenem Einkommen bzw.

\footnotetext{
1 Dass die geplante Reform der Koalition die "großen « Probleme der Pflege nicht löst, habe ich vor kurzem in der Sozialen Sicherheit, Ausgabe 11/2011 dargelegt: »Bundesregierung verabschiedet Skizzen eines Reförmchens - Riesiger Handlungsdruck bleibt «, Seite $384 \mathrm{ff}$.
} 
Vermögen mindestens die Kosten seines Lebensunterhalts zu tragen hat, so wie sie ohne Eintritt der Pflegebedürftigkeit angefallen wären. Damit liegt auf der Hand, dass die Pflegeversicherung auf eine funktionierende Alterssicherung angewiesen ist.

Die Pflegeversicherung wurde daher schon bei ihrer Einführung als »Teilkaskoversicherung" konzipiert. Die neue Versicherung sollte als ZuschussLeistung vor allem die professionellen Pflegeleistungen finanzieren. Zusam-

\section{Unsere Pflegeversicherung ist auf eine funktionierende Alterssicherung angewiesen.}

tersarmut mitverursacht sein. Jedenfalls gibt der gesamtwirtschaftliche Sachverständigenrat (SVR) in seinem Jahresgutachten 2011/2012 der »Prävention von Altersarmut « inzwischen die erste rentenpolitische Priorität ${ }^{3}$. Zwar hält der Rat die Altersarmut zur Zeit noch nicht für ein "gesellschaftlich relevantes Problem ${ }^{4}$. Aber der Anstieg der Empfängerzahl von »Grundsicherung im Alter" stimmt ihn bedenklich. Die Zahl der Empfänger erreicht 2010 mit 410.000 Personen ihren Höchststand. Sie stieg damit gegenüber dem Jahr 2003, dem Jahr der Einführung bzw. Neukonzeption dieses Teils der Sozialhilfe, um rund 60 Prozent, "während die Anzahl der Personen über 64 Jahre im gleichen Zeitraum lediglich um 13,4 Prozent

men mit den anderen Alterseinkommen sollte sie den meisten Pflegebedürftigen ermöglichen, ohne Rückgriff auf die Sozialhilfe auszukommen. Daraus ergibt sich auch, wie wichtig die Stabilität des Rentensystems im Verhältnis zur Pflegeversicherung ist. Nur bei sicheren und ausreichenden Alterseinkommen kann das Konzept der Pflegeversicherung aufgehen.

Dabei wurden diese Ziele nicht nur bei der Einführung sondern immer wieder kritisiert. Es ginge vor allem um die Entlastung der Kommunen als Sozialhilfeträger bzw. um die Entlastung der Kindergeneration von Pflegelasten für ihre Eltern. Böse Zungen haben die Pflegeversicherung daher auch als »Erbenschutzprogramm« bezeichnet.

Schon bald nach ihrer Einführung stieg erneut die Zahl der Fälle, in denen die Sozialhilfe doch wieder einspringen musste. Das hing mit der Fixierung der Leistungssätze zusammen, die durch die Inflation schleichend entwertet wurden. So bezogen etwa im Laufe des Jahres 2008 rund 397.000 Personen »Hilfe zur Pflege«, die ergänzend zu den Leistungen der Pflegeversicherung gezahlt wird. Das entsprach gegenüber 1998 einem Anstieg von 37 Prozent $^{2}$. 72 Prozent dieser Sozialhilfeempfänger (rund 290.000) lebten in stationären Einrichtungen.

Der erneute Anstieg der Kofinanzierung der Pflegebedürftigen durch die Sozialhilfe könnte aber auch durch das allmählich anrollende Problem der Al- zunahm. «5 Während der SVR für die Gegenwart noch darüber spekuliert, ob dieser Anstieg der Sozialhilfeempfänger vielleicht etwas mit dem Abbau der »versteckten Armut« zu tun hat, sieht er jedenfalls für die Zukunft ohne Zweifel das Risiko der Altersarmut. Als ursächliche Entwicklungen sieht er die Situation auf dem Arbeitsmarkt, insbesondere die Verfestigung von Langzeitarbeitslosigkeit, und die »Aufspreizung der Entlohnungsstruktur am unteren Ende der Lohnskala «. ${ }^{6}$ Außerdem trügen die Veränderung der Erwerbstätigständigkeit) und die Zunahme bei der Teilzeitbeschäftigung zu den künftig erwarteten Problemen bei.

Als Lösungsmodelle diskutiert der SVR eine obligatorische Versicherungspflicht für die Selbständigen ${ }^{7}$ und sympathisiert mit der von der Bundesregierung vorgeschlagenen "Zuschussrente « ${ }^{8}$ Bei diesem Konzept der (steuerfinanzierten?) Ergänzung der gesetzlichen Rentenansprüche ist allerdings maximal ein Zahlbetrag von 850 Euro im Monat erreichbar. Damit dürfte jedem klar sein, dass auch die Zuschussrente die Betroffenen im Fall der Pflegebedürftigkeit kaum vor der Sozialhilfe retten wird.

Viel dramatischer bewertet Winfried Schmähl die Lage9. Für den früheren Vorsitzenden des Sozialbeirats tickt bei der Alterssicherung eine "gesellschaftspolitische Zeitbombe ${ }^{10}{ }^{10}$ Er zeigt nämlich, dass es in Zukunft selbst für Durchschnittsverdiener immer schwerer keitsstruktur (Zunahme prekärer Selb- werden wird, mit der gesetzlichen Rentenversicherung wenigstens das Grundsicherungsniveau zu erreichen. Der Abschied vom Konzept der lebensstandardsichernden gesetzlichen Rente habe dazu geführt, dass ein Durchschnittsverdiener in Zukunft »erst nach 35 Beitragsjahren auf eine GRV-Rente in Höhe der Grundsicherung (also des Sozialhilfeniveaus) kommen (würde), wenn er mit 67 in Rente geht.« Er weist auch einmal mehr - darauf hin, dass - wegen der steuerlichen Förderungsanreize - die Privatvorsorge vor allem die mittleren und höheren Einkommen begünstigt, ${ }^{11}$ was natürlich auch für entsprechende »Riester-Modelle« einer privaten PflegeZusatzversicherung gelten würde. Für die niedrigen Einkommen ist - angesichts der bevorstehenden Gesamtkonstellation - dagegen zu befürchten, dass die Vorsorgebereitschaft abnehmen wird, denn die Frage drängt sich gerade für diese Gruppen auf: Lohnt sich (private) Vorsorge überhaupt? ${ }^{12}$

Dabei weist Schmähl explizit auf den Zusammenhang der Sozialversicherungszweige hin: »Im Zusammenspiel mit den Veränderungen in der Alterssicherung droht insbesondere für Pflegebedürftige zunehmend die Gefahr der Sozialhilfeabhängigkeit ... . Alterssicherungspolitik sollte folglich nicht nur die Entwicklung in den Alterssicherungssystemen und bei der Besteuerung berücksichtigen, sondern auch die Entwicklung insbesondere der Leistungen im Gesundheitswesen bei Krankheit und bei Pflegebedürftigkeit. «13 Letzteres gilt natürlich auch umgekehrt für die Pflegepolitik in Richtung auf die Sicherung der Alterseinkommen.

\footnotetext{
2 vgl. Statistisches Bundesamt: Statistik der Sozialhilfe. Hilfe zur Pflege 20078, Wiesbaden 2010, S. 3

3 Sachverständigenrat Jahresgutachten 2011/2012, S. 309.

4 a.a.O. S. 313

5 a.a.O. S. 314

6 a.a.O. S. $314 \mathrm{f}$.

7 a.a.O. S. 318

8 a.a.O. S. 322

9 Winfried Schmähl: »Der Paradigmawechsel in der Alterssicherungspolitik: Die RiesterReform von 2001 - Entscheidungen, Begründungen, Folgen «, in Soziale Sicherheit 12/2011, S. $405 \mathrm{ff}$

10 a.a.O. S. 414

11 a.a.O. S. S. 409

12 vgl. a.a.O. S. 412

13 a.a.O. S. 410
} 


\section{Krankenversicherung und Pflege}

Die Tatsache, dass Pflege- und Krankenversicherung in ein und derselben Institution verwaltet werden, ist noch lange keine Garantie dafür, dass beide Systeme gut zusammenarbeiten. Ganz

\section{Dass Pflege- und Kranken- versicherung in ein und derselben Institution verwaltet werden, ist noch lange keine Garantie dafür, dass beide Systeme gut zusammenarbeiten.}

im Gegensatz zu dieser eher naiven Erwartung gibt es viele Fehlstellen und Brüche, die vor allem etwas mit den Bewegungsgesetzen der Krankenkassen zu tun haben, die sich immer wieder gegen die Interessen der Pflegeversicherung durchsetzen. Worum geht es:

- Die Pflegebedürftigkeit und ihre jeweilige Einstufung ist - ganz unabhängig von dem grade geltenden »Pflegebegriff« - weniger schicksalhaft und endgültig als oft angenommen wird. Die Möglichkeiten der Prävention von Pflege, der geriatrischen Rehabilitation und des systematischen Hinausschiebens einer Verschlechterung des Pflegezustandes werden zu wenig genutzt. Die diesen Zielen entsprechenden Leistungen sind jedoch noch zu wenig definiert und jedenfalls nicht in routinemäßige Prozesse eingebunden. Auch aus diesen Gründen wird hier weniger getan, als möglich und sinnvoll wäre. Die Gesundheitsförderung für Hochbetagte oder geriatrische Rehabilitation unterbleiben oft auch deshalb, weil die GKV hier Kostenträger ist. Die erfolgreiche Prävention von Pflegebedürftigkeit bzw. die Abwendung eines erhöhten Pflegebedarfs ist nach der ökonomischen Logik einer wettbewerblich strukturierten und am morbiditätsorientierten RSA orientierten GKV ökonomisch kein sinnvoller Anreiz $^{14}$. Die Verwaltung und Steuerung der Pflegeversicherung als Einheitsversicherung durch Institutionen, die wettbewerbliche Eigeninteressen haben, stellt sich hier als grundsätzliches Problem heraus.

- Das konkretisiert sich auch regelmäßig an Zuständigkeits- und Abgrenzungsproblemen. Bekannt sind die schließlich vor den Gerichten entschiedenen Streitereien um die Finanzierung von »Pflegebetten « und anderen Pflegehilfsmitteln. Dass die medizinische Behandlungspflege « (immer noch) in der Zuständigkeit der Pflegeversicherung liegt, ist eine politische Entscheidung, die gerade im Zusammenhang mit der aktuellen »Pflegereform« wieder einmal in Frage gestellt worden ist. Besonders plastisch wird der Zusammenhang an der durch das Pflegeweiterentwicklungsgesetz eingeführten Möglichkeit ( $\mathbb{S} 92 \mathrm{~b}$ SGB XI) zur Beteiligung der Pflegekassen und von Pflegeeinrichtungen an der Integrierten Versorgung nach \140b Abs. 1 SGB V. So gut gemeint diese Regelung ist, so wenig ist sie bisher mit Leben erfüllt worden. Die Krankenkassen haben einfach nicht genügend Interesse, in solche Projekte $\mathrm{zu}$ investieren.

- Die medizinische Versorgung und ihre Qualität für die Pflegebedürftigen wird immer wieder als defizitär kritisiert, obwohl es hier um eine genuine Aufgabe und Zuständigkeit der Krankenversicherung geht. Vor allem die fachärztliche Versorgung in den Heimen steht in der Diskussion. Mit der seit 2008 geltenden Möglichkeit der Ermächtigung von »Heimärzten« (nach $\mathbb{S} 119 \mathrm{~b}$ SGB V) und der im GKV-Versorgungsstrukturgesetz in $\mathbb{} 87$ Abs. $2 \mathrm{i}$ (neu) vorgesehenen "Abrechnungsziffer für Hausbesuche« von Zahnärzten bei Pflegebedürftigen sind Schritte in die richtige Richtung unternommen worden. Sie reichen aber noch längst nicht aus, was in den aktuellen »Eckpunkten« der Bundesregierung zur Pflegereform auch anerkannt wird. Welche Verbesserungen konkret daraus folgen, bleibt abzuwarten.

- Ein weiter brennendes Problem ist das Fallmanagement bei Multimorbidität. Millionen ältere Menschen erhalten ungeeignete und zu viele Medikamente $^{15}$. »In Deutschland sind rund zehn Prozent der Krankenhauseinweisungen bei Menschen über 65 Jahren das Ergebnis von falschen oder zu hoch dosierten Medikamenten «, sagt Prof. Gerd Glaeske vom Zentrum für Sozialpolitik der Universität Bremen ${ }^{16}$. 5,5 Millionen Menschen sind Risiken durch Polymedikation ausgesetzt ${ }^{17}$. Auch die Probleme einer sachgemäßen Medikamenteneinnahme werden immer wieder diskutiert; Verbesserungsversuche z. B. mit diversen Verblisterungs-Verfahren sind im Gang. Das Arzneimittelmanagement für ältere Menschen wäre daher ein vielversprechender Ansatz zur Prävention von Pflegebedürftigkeit und zur Verbesserung der gesundheitlichen Lage der Pflegebedürftigen.

Die Beispiele mögen genügen. Die Schnittstelle von GKV und Pflege bedarf der »sektorübergreifenden « Integration und Flexibilisierung mindestens in dem gleichen Maße wie die Schnittstelle von ambulant und stationär (sowohl in der Gesundheitsversorgung wie auch in der Pflege!). Die unterschiedlichen Organisationsinteressen und Finanzierungslo-

\section{Die Schnittstelle von GKV und Pflege bedarf der sektor- übergreifenden Integration.}

giken stehen dem jedoch entgegen. Hier sind Kreativität und neue institutionelle Arrangements gefragt, bei denen auch ein kommunales Engagement für Gesundheitspolitik und Pflegeversorgung einen neuen Stellenwert gewinnen muss.

14 Dass nach den Eckpunkten zur Pflegereform, die das Bundeskabinett am 16.11.2011 beschlossen hat, die Rehabilitation gestärkt werden soll und jeder Pflegebedürftige ein "Gutachten über seine individuelle Rehabilitationsfähigkeit« erhalten soll, ist sicher ein richtiger Schritt. Man darf aber gespannt sein, welche Veränderungen der Praxis tatsächlich daraus folgen ...

15 vgl. Thürmann, Petra A. et al.: »Arzneimittelversorgung älterer Patienten «, in Günster, Christian, Joachim Klose, Norbert Schmacke (Hg.): »Versorgungsreport 2012«, Stuttgart 2012

16 Ruhr Nachrichten vom 23.12.2011

17 Thomas Rottschäfer: »Härtetest für den Generationenvertrag «, in Gesundheit und Gesellschaft 11/11, Seite 34 


\section{Wohnungs-, Verkehrs- und Kommunalpolitik}

Ein wichtiges Element des »neuen « Pflegebegriffs ist die Einbeziehung des Aspekts der sozialen Integration der Pflegebedürftigen. Es wäre jedoch eine Überforderung der Pflegeversicherung, sie alleine für die »Soziale Integration" der Pflegebedürftigen in die Pflicht zu nehmen. Die Frage ist, wie weit diese Aufgabe überhaupt zur Sozialversicherung gehört und wieweit andere gesellschaftliche Institutionen dabei mitwirken müssen. Im Rahmen diese Aufsatzes können dazu nur wenige Punkte angesprochen werden.

- Pfarrer Dr. Jürgen Gohde, der Vorsitzende des Beirats zum »Pflegebegriff « erklärt dazu: »Die Pflegeversicherung ist nicht der einzige Baustein. Eine große Herausforderung ist Infrastruktur der Pflege. Stichworte sind hier etwa altersgerechtes Wohnen, nachbarschaftliche Arrangements, Koordinationen zwischen hauptamtlichen Pflegekräften und Ehrenamtlichen, verbesserte Pflegeberatung, bessere Rahmenbedingungen für den Pflegeberuf sowie die ... Entlastung der Angehörigen und die Förderung der Potenziale älterer Menschen. «18 Und weiter fordert er »vernetzte Versorgungsformen, die auf kommunaler Ebene Verantwortung übernehmen«. - Ein wichtiger Anstoß ist dabei der altersgerechte Umbau von Wohnungen. Das vom Bundesministerium für Verkehr, Bau und Stadtentwicklung (BMVBS) mit Haushaltsmitteln geförderte und in 2009 aufgelegte Programm »Altersgerecht Umbauen« ist jedoch Ende 2011 in seiner bisherigen Form ausgelaufen. Im Haushalt der Bundesregierung für 2012 sind - entgegen der massiven Forderungen der Fachleute - keine weiteren Mittel vorgesehen. Allerdings wird die KfW-Bank das Programm ab Januar 2012 in der »Darlehensvariante « als Eigenprogramm fortführen. Das Programm wird künftig aus KfWMitteln im Zins verbilligt. Dennoch wird die KfW die bisherige Subventionsleistung des Bundes lediglich teilweise kompensieren können. Bis Ende September 2011 wurde der Umbau von rund 59.000 Wohnungen mit einem Kreditvolumen von über einer halben Milliarde Euro gefördert. Der Bedarf geht aber weit darüber hinaus. In Deutschland gibt es z.Z. gerade einmal rund eine halbe Million weitgehend altersgerechter Wohnungen. Nach einer Berechnung des Kuratoriums Deutsche Altershilfe müssen bis 2020 mindestens 2,5 Millionen Wohnungen hinzukommen.

- Minister Ramsauer (BMVBS) nennt selbst ein paar Beispiele, um was es geht: »Der Abbau von Barrieren beginnt spätestens an der Wohnungstür. Sie sollte sich ohne großen Kraftaufwand öffnen lassen. Die Räume müssen ohne Schwellen zu erreichen sein. Im Bad braucht es Haltegriffe und eine bodengleiche Dusche. $\ll^{19}$ Das sind typischerweise auch die Leistungen, die die Pflegeversicherung zur "Verbesserung des individuellen Wohnumfeldes« der Pflegebedürftigen nach $\mathbb{S} 40$ Abs. 4 SGB XI unterstützen kann. Die eigentlich dahinter stehende Aufgabe geht aber weit über die »klassischen" Sozialmaßnahmen hinaus. Die SPD fordert z. B. in ihrem gesundheitspolitischen Leitantrag bei ihrem Berliner Parteitag 2011, den maximalen Förderbetrag für diese Leistung in Höhe von 2.557 Euro je Maßnahme praktisch zu verdoppeln. Das ist gut gemeint, kommt aber den Betroffenen möglicherweise nicht in dem erwünschten Umfang zu Gute. Denn erstens zeigt die Erfahrung, dass bei Maßnahmen, die zu Lasten der Sozialversicherung abgerechnet werden, die üblichen Marktpreise entsprechender Leistungen in der Regel weit überschritten werden. Zweitens liegt auf der Hand, dass solche Baumaßnahmen sehr viel effektiver und billiger durch z. B. Wohnungsbaugesellschaften durchgeführt werden könnten, als im Rahmen individueller Einzelmaßnahmen. Hier liegt eine Aufgabe für das kommunale Quartiersmanagement und vor allem die öffentlichen Wohnungsunternehmen, die bei solchen Maßnahmen mit gutem Beispiel vorangehen müssten.

- Diese Gesichtspunkte können für weitere Lebensbereiche durchdekliniert werden, etwa für Mobilität und Verkehrspolitik, IT-Infrastruk- tur etc. Dabei geht es nicht nur um Pflegeinfrastruktur, sondern Versorgungsinfrastruktur ganz generell. Hier müssen im Hinblick auf die Alterung unserer Gesellschaft mittelfristig Aufgaben, die bislang bei den Sozialversicherungsträgern angesiedelt waren, schrittweise auf die Kommunen zurückverlagert und in deren Daseinsvorsorge integriert werden. In diesem Sinne wird hier exemplarisch das "Netzwerk: Soziales neu gestalten « zitiert. Es fordert eine Rekommunalisierung der Pflegepolitik und in diesem Zusammenhang eine Neubestimmung und Revitalisierung des Subsidiaritätsprinzips ${ }^{20}$. Kritisch wird gesehen, dass sich die Kommunen mit der Einführung der Pflegeversicherung aus der Infrastrukturentwicklung und z. B. der Planung von stationären Einrichtungen weitge- 
MDK-Begutachtungsstatistik

$\begin{array}{ll}\text { Gutachterliche Empfehlung einer Pflegestufe (BA12a) } \\ \text { Berichtszeitraum: } & 2010 \\ \text { Leistungsart: } & \text { ambulant }\end{array}$

\begin{tabular}{|c|c|c|c|c|c|}
\hline \multirow{2}{*}{$\begin{array}{l}\text { MDK/ } \\
\text { Sonderdienste }\end{array}$} & \multirow{2}{*}{$\begin{array}{l}\text { Durch- } \\
\text { geführte } \\
\text { Erstbegut- } \\
\text { achtungen }\end{array}$} & \multicolumn{4}{|c|}{ davon i.v.H. } \\
\hline & & $\begin{array}{l}\text { nicht } \\
\text { pflege- } \\
\text { bed. }\end{array}$ & $\begin{array}{l}\text { Pflege- } \\
\text { stufe I }\end{array}$ & $\begin{array}{l}\text { Pflege- } \\
\text { stufe II }\end{array}$ & $\begin{array}{l}\text { Pflege- } \\
\text { stufe III }\end{array}$ \\
\hline $\begin{array}{l}\text { Baden-Württem- } \\
\text { berg }\end{array}$ & 64.995 & 29,1 & 54,4 & 14,5 & 2,0 \\
\hline Bayern & 80.846 & 36,0 & 46,3 & 13,0 & 4,7 \\
\hline $\begin{array}{l}\text { Berlin-Branden- } \\
\text { burg }\end{array}$ & 50.796 & 37,7 & 51,2 & 9,6 & 1,5 \\
\hline Bremen & 5.393 & 34,2 & 52,5 & 11,1 & 2,1 \\
\hline Hessen & 46.684 & 31,3 & 52,6 & 12,7 & 3,3 \\
\hline $\begin{array}{l}\text { Mecklenburg- } \\
\text { Vorpommern }\end{array}$ & 17.036 & 25,5 & 56,7 & 15,4 & 2,4 \\
\hline Niedersachsen & 54.297 & 32,9 & 57,4 & 8,6 & 1,1 \\
\hline Nord & 23.056 & 46,6 & 45,9 & 6,7 & 0,7 \\
\hline Nordrhein & 67.288 & 32,4 & 53,9 & 12,1 & 1,7 \\
\hline Rheinland-Pfalz & 34.639 & 37,7 & 43,8 & 17,2 & 1,3 \\
\hline Saarland & 7.642 & 37,2 & 43,5 & 12,3 & 6,9 \\
\hline Sachsen & 39.592 & 38,1 & 45,9 & 12,5 & 3,5 \\
\hline Sachsen-Anhalt & 23.447 & 35,0 & 49,0 & 11,9 & 4,1 \\
\hline Thüringen & 21.384 & 32,1 & 47,4 & 14,0 & 6,5 \\
\hline Westfalen-Lippe & 63.625 & 38,5 & 46,2 & 13,8 & 1,4 \\
\hline Summe & 600.720 & 34,8 & 50,2 & 12,5 & 2,6 \\
\hline Knappschaft & 45.060 & 26,0 & 57,0 & 13,8 & 3,1 \\
\hline $\begin{array}{l}\text { Bundeseisen- } \\
\text { bahnvermögen }\end{array}$ & 9.417 & 24,0 & 61,4 & 12,6 & 2,0 \\
\hline Gesamt & 655.197 & 34,0 & 50,8 & 12,6 & 2,6 \\
\hline
\end{tabular}

Begutachtungsstatistik des MDS 2010, Stand 17.07.2011, Seite BA12a

öffnet, die Sektorgrenzen zwischen ambulant und stationär in der Pflege zu flexibilisieren und »neue gemischte nicht-stationäre Versorgungskonzepte $«$ zu realisieren ${ }^{22}$. Und so weiter.

Um den Bezug zur aktuellen Pflegereform wiederherzustellen: In den $»$ Eckpunkten" der Regierungskoalition werden verschiedene Leistungsverbesserungen angekündigt. Das geht von der Flexibilisierung der Pflegeleistungen über die Förderung neuer ambulanter
Wohnformen, das Initiativprogramm für den Aufbau von Wohngruppen bis zur Entbürokratisierung der Pflegeversicherung. Einen durchschlagenden Erfolg können solche Maßnahmen aber nur haben, wenn sie sich nicht allein auf die Regelungen des SGB XI beschränken, sondern andere Politikfelder mit einbeziehen. »Pflege « ist somit nicht nur ein Thema für die Gesundheits- und Sozialpolitik, sondern eines der zentralen $\mathrm{Zu}$ kunftsthemen der Gesellschaftspolitik insgesamt.

\section{Regionale Aspekte}

Nach alledem liegt die Bedeutung der regionalen bzw. kommunalen (Pflege-) Infrastruktur auf der Hand. Über regionale Unterschiede beim Thema Pflege ist jedoch relativ wenig bekannt, bzw. die Unterschiede werden kaum diskutiert. Die vorhandenen Statistiken bewegen sich auch fast nur auf der Ebene der Bundesländer bzw. der Medizinischen Dienste der Krankenkassen (MDK). Die

\section{Über regionale Unterschiede beim Thema Pflege ist wenig bekannt.}

eigentlich spannenden Unterschiede liegen jedoch vermutlich unterhalb dieser Ebene. Aber auch die verfügbaren $\mathrm{Da}$ ten vermitteln zumindest eine Ahnung davon, dass sich z. B. die Risiken von Pflegebedürftigkeit regional stark unterscheiden, dass die Bereitschaft und die Möglichkeit, Pflegeaufgaben zu übernehmen regional recht unterschiedlich sind und schließlich auch die Begutachtungspraxis für die Pflege keineswegs einheitlich ist.

So liegen die Pflegequoten ${ }^{23}$ nach der Pflegestatistik 2009 des Statistischen Bundesamtes im Ländervergleich zwischen 2,29 Prozent (Baden-Württemberg) und 3,72 Prozent (MecklenburgVorpommern $)^{24}$. In einer Sonderauswertung des Statistischen Bundesamtes für die Pflegestatistik des Jahres 2007 auf der Kreisebene zeigen sich noch massivere Unterschiede: "Auf Ebene der Kreise lag der höchste Anteil mit 5,0 \% in der kreisfreien Stadt Passau in Bayern vor." Demgegenüber lag der geringste Anteil der Pflegebedürftigen in den bayerischen Landkreisen Freising und Erding (beide 1,5\%). »Dem folgten die badenwürttembergischen Landkreise Böblingen und Ludwigsburg (jeweils 1,7 \%). Die beiden kreisfreien Städte mit dem geringsten Anteil lagen in Baden-Württemberg bzw. Bayern: Stuttgart (1,8 \%)

22 a.a.O. Seite 23

23 Anteil der Pflegebedürftigen an der Wohnbevölkerung

24 Statistisches Bundesamt: »Pflegestatistik 2009 - Ländervergleich «, erschienen im April 2011, Seite 8 
MDK-Begutachtungsstatistik

Gutachterliche Empfehlung einer Pflegestufe (BS12a)

Berichtszeitraum:

2010

Leistungsart:

vollstationär

\begin{tabular}{|c|c|c|c|c|c|}
\hline \multirow{2}{*}{$\begin{array}{l}\text { MDK/ } \\
\text { Sonderdienste }\end{array}$} & \multirow{2}{*}{$\begin{array}{l}\text { Durch- } \\
\text { geführte } \\
\text { Erstbegut- } \\
\text { achtungen }\end{array}$} & \multicolumn{4}{|c|}{ davon i.v.H. } \\
\hline & & $\begin{array}{l}\text { nicht } \\
\text { pflege- } \\
\text { bed. }\end{array}$ & $\begin{array}{l}\text { Pflege- } \\
\text { stufe I }\end{array}$ & $\begin{array}{l}\text { Pflege- } \\
\text { stufe II }\end{array}$ & $\begin{array}{l}\text { Pflege- } \\
\text { stufe III }\end{array}$ \\
\hline $\begin{array}{l}\text { Baden-Württem- } \\
\text { berg }\end{array}$ & 18.397 & 16,6 & 51,4 & 28,2 & 3,8 \\
\hline Bayern & 21.152 & 19,7 & 44,2 & 26,7 & 9,3 \\
\hline $\begin{array}{l}\text { Berlin-Branden- } \\
\text { burg }\end{array}$ & 4.730 & 16,9 & 51,6 & 24,5 & 7,0 \\
\hline Bremen & 637 & 11,9 & 59,0 & 26,7 & 2,4 \\
\hline Hessen & 6.468 & 13,3 & 45,1 & 30,7 & 10,9 \\
\hline $\begin{array}{l}\text { Mecklenburg- } \\
\text { Vorpommern }\end{array}$ & 1.960 & 4,7 & 55,3 & 38,7 & 1,3 \\
\hline Niedersachsen & 7.477 & 18,4 & 57,7 & 21,9 & 2,0 \\
\hline Nord & 3.429 & 36,6 & 49,5 & 12,0 & 1,9 \\
\hline Nordrhein & 15.364 & 12,7 & 51,2 & 30,2 & 5,9 \\
\hline Rheinland-Pfalz & 6.832 & 24,6 & 44,0 & 28,9 & 2,5 \\
\hline Saarland & 2.080 & 29,1 & 40,3 & 21,0 & 9,6 \\
\hline Sachsen & 6.950 & 10,1 & 44,8 & 34,5 & 10,5 \\
\hline Sachsen-Anhalt & 4.202 & 14,2 & 43,8 & 31,6 & 10,4 \\
\hline Thüringen & 2.969 & 5,6 & 46,2 & 38,5 & 9,7 \\
\hline Westfalen-Lippe & 11.543 & 17,6 & 46,0 & 32,5 & 3,9 \\
\hline Summe & 114.190 & 17,0 & 48,2 & 28,6 & 6,3 \\
\hline Knappschaft & 13.140 & 8,9 & 57,1 & 28,5 & 5,5 \\
\hline $\begin{array}{l}\text { Bundeseisen- } \\
\text { bahnvermögen }\end{array}$ & 1.124 & 10,9 & 50,3 & 32,1 & 6,7 \\
\hline Gesamt & 128.454 & 16,1 & 49,1 & 28,6 & 6,2 \\
\hline
\end{tabular}

Begutachtungsstatistik des MDS 2010, Stand 17.07.2011, Seite BS12a

und München (1,9\%).« «5

Deutliche Unterschiede gibt es auch bei den Quoten der "pflegenahen « Jahrgänge. So variiert die Pflegequote bei den 75 - 85 jährigen Frauen zwischen 12,9 (Baden-Württemberg) und 21,0 (Mecklenburg-Vorpommern). Bei den 85 - 90 -jährigen Frauen reicht die Spanne von 35,4 (Baden-Württemberg) bis 54,0 Prozent (Mecklenburg-Vorpommern) und so weiter ${ }^{26}$.

Auch die Begutachtungsergebnisse unterscheiden sich. So führen beispielswei- der Antragstellung nicht nur die Pflegebedürftigen selbst eine Rolle. Auch die Einschätzungen der niedergelassenen Ärzte unterscheiden sich offenbar zwischen den Regionen, denn sie sind gewöhnlich die ersten Ratgeber der Versicherten in Sachen Pflegebedürftigkeit und wirken im allgemeinen bei der Antragstellung für das Begutachtungsverfahren mit.

Schließlich weisen auch die MDKEmpfehlungen für »Härtefälle« erhebliche Unterschiede auf. Sie liegen im ambulanten Bereich zwischen 0,6 Prozent in Bayern und 3,2 Prozent in Bremen ${ }^{29}$. Für die Härtefälle im vollstationären Bereich liegen die Quoten zwischen 0,7 Prozent in Rheinland-Pfalz und 15,0 Prozent in Berlin-Brandenburg ${ }^{30}$. Bemerkenswert ist in diesem Zusammenhang, dass sich die Einstufung in die drei Pflegestufen, wenn Pflegebedürftigkeit erst einmal anerkannt ist, bei allen MDKs ziemlich ähnlich darstellt: Mehr als die Hälfte der Pflegebedürftigen bekommt die Stufe I. Die entsprechenden Quoten variieren "nur" zwischen 51,0 (Bayern) und 57,4 (Mecklenburg-Vorpommern). Bei der Vergabe der Pflegstufe III gibt es dann wieder etwas größere Unterschiede: Am seltensten wurde sie mit 9,4 Prozent in Mecklenburg-Vorpommern vergeben, in Bayern hatte sie dagegen immerhin einen Anteil von 15,5 Prozent ${ }^{31}$. Diese Ergebnisse lagen in der Frühzeit der Pflegeversicherung ebenfalls weiter auseinander. Hier haben sich offenbar die Abstimmungsbemühungen der einzelnen MDKs inzwischen gelohnt.

Trotzdem bleiben die Unterschiede bedenklich. Beispielsweise schwankt die Quote der Begutachtungen mit Hausbesuch bei den ambulanten Begutachtungen zwischen 75 und 99 Prozent; dementsprechend der Anteil der Begutachtungen "nach Aktenlage»

25 Statistisches Bundesamt: »Pflegestatistik 2007 - Kreisvergleich «, erschienen im Februar 2010, Seite 6

26 Statistisches Bundesamt: »Pflegestatistik 2009 - Ländervergleich «, erschienen im April 2011, Seite 8

27 Medizinischer Dienst des GKV Spitzenverbandes (MDS): Begutachtungsstatistik 2010 für den Bereich der sozialen Pflegeversicherung, Stand: 17.07.2011, Seite BA12a

28 ebenda

29 a.a.O. Seite BA13

30 a.a.O. Seite BS13

31 Statistisches Bundesamt: »Pflegestatistik 2009 - Ländervergleich «, erschienen im April 2011, Seite 10 
zwischen 25 und 0,3 Prozent ${ }^{32}$. Noch krassere »Methoden «-Unterschiede gibt es bei der Begutachtung im stationären Bereich $^{33}$. Das gilt auch für die Betei-

\section{Es gibt Methoden- Unterschiede bei der Begutachtung.}

ligung der MDK-Ärzte an den Begutachtungen und den Anteilen eigener und externer Gutachter. So gibt es für den vollstationären Bereich beispielsweise in Baden-Württemberg überhaupt keine Beteiligung externer Gutachter, im Saarland liegt deren Quote jedoch bei 99 Prozent $^{34}$. Und so weiter.

Diese Zahlen sind nicht auf die jeweilige Bevölkerung des Bundeslandes bzw. des MDK-Bezirks standardisiert und schon gar nicht morbiditätsadjustiert. Hier ist sicher noch viel zu tun und reichlich Forschungsbedarf. Angesichts der Größe und Vielfalt der Unterschiede drängt sich jedoch die Vermutung auf, dass sie sich nicht nur durch demographische und Morbiditätsunterschiede erklären lassen. Das wäre immerhin sozialpolitisch von erheblicher Bedeutung, weil das Leistungsversprechen der Pflegeversicherung bundesweit einheitlich ist, Leistungsunterschiede etwa durch wettbewerbliche Verträge (wie in der GKV) keineswegs beabsichtigt sind und drittens die Pflegeversicherung nicht nur mit einem einheitlichen Beitragssatz, sondern auch mit einem vollständigen Ausgabenausgleich über alle Träger funktioniert.

Erhebliche regionale Unterschiede zeigen sich auch bei der Organisation der Pflege. Im Bundesdurchschnitt liegt der Anteil der stationären Pflege bei ca. 31 Prozent. Die stärkste Bedeutung hat die stationäre Pflege aber in SchleswigHolstein - $41 \%$ aller Pflegebedürftigen wurden dort vollstationär versorgt. »In Brandenburg und in Hessen wurden hingegen nur ca. $25 \%$ der Pflegebedürftigen in Heimen ... betreut. «35

»Die Versorgung durch ambulante Pflegedienste hat ein hohes Gewicht in Hamburg und Brandenburg (jeweils $30 \%)$ sowie in Sachsen $(28 \%)-$ in Brandenburg wurden ... mehr Pflegebedürftige durch ambulante Pflegedienste als durch Pflegeheime versorgt.

Kreiskarte: Anteil der Pflegebedürftigen an der Bevölkerung insgesamt Jahresende 2007-

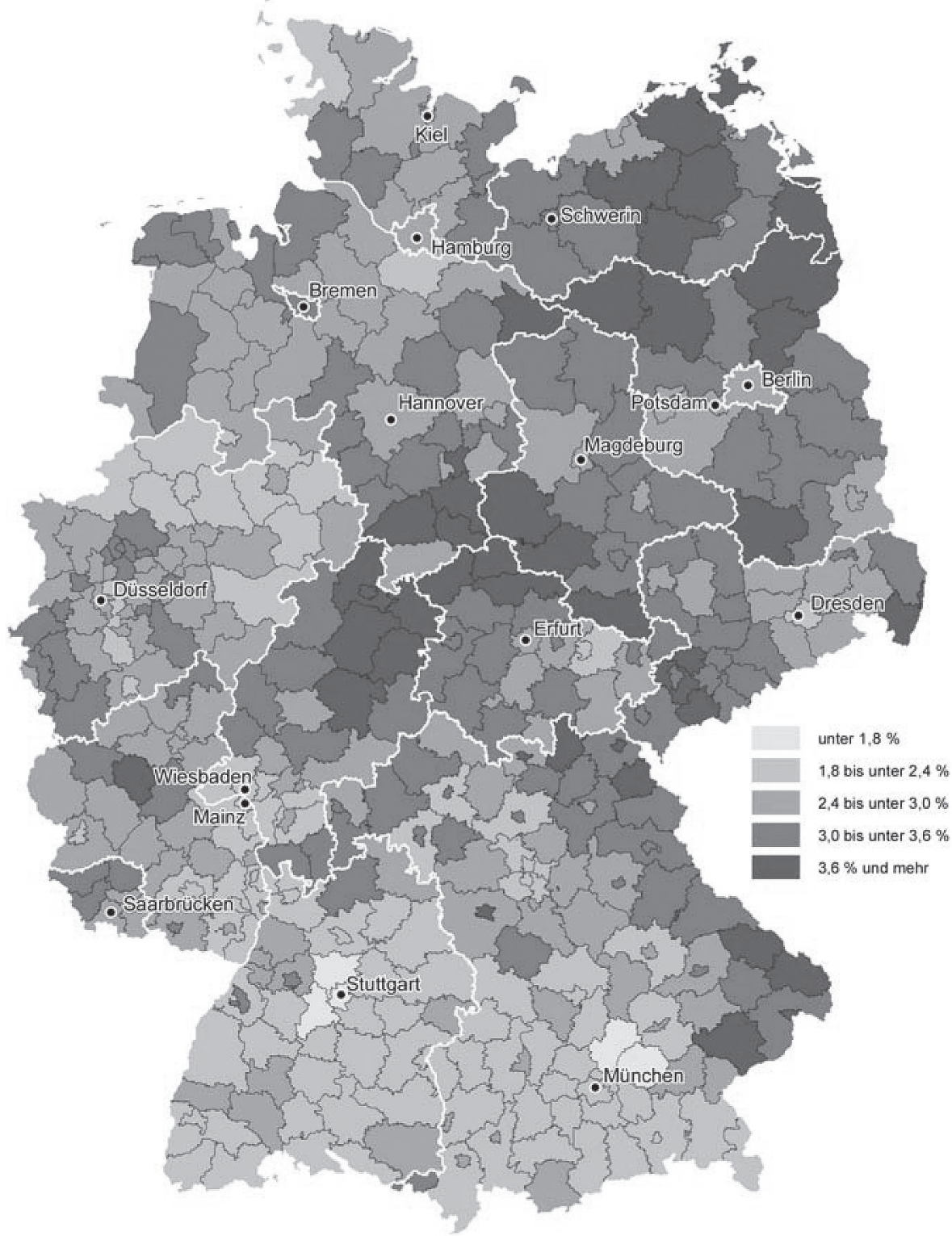

Quelle: Statistische Ämter des Bundes und der Länder @ Bundesamt für Kartografie und Geodäsie (www.bkg.bund.de)

Statistische Ämter des Bundes und der Länder, Pflegestatistik 2007-Kreisvergleich, Seite 7

Bundesweit betrug der Anteil der ambulanten Pflegedienste $24 \%$. Die Betreuung allein durch Angehörige erfolgt insbesondere in Hessen (Anteil: $54 \%$ ), Rheinland-Pfalz (50 \%) und Thüringen (49\%). Die Bedeutung der Pflege durch Angehörige ist - auch infolge der starken Bedeutung der Pflege im Heim - in Schleswig-Holstein relativ gering (38 $\%)$. Die niedrigen Werte in Sachsen (39 $\%)$ und in Hamburg (39\%) sind auch bedingt durch den hohen Anteil der ambulanten Pflegedienste an der Versorgung. Im Bundesdurchschnitt wurden $46 \%$ der Pflegebedürftigen von Ange- hörigen versorgt. « ${ }^{36}$

Auf der Ebene »der Kreise hatte die vollstationäre Dauerpflege das größte Gewicht in der bayerischen Stadt Landshut mit einem Anteil von 54,9\%, ge-

32 Medizinischer Dienst des GKV Spitzenverbandes (MDS): Begutachtungsstatistik 2010 für den Bereich der sozialen Pflegeversicherung, Stand: 17.07.2011, Seite BA20a

33 a.a.O. Seite BS20a

34 a.a.O. Seite BS30

35 Statistisches Bundesamt: »Pflegestatistik 2009 - Ländervergleich «, erschienen im April 2011, Seite 11

36 ebenda 


\section{Pflege durch Angehörige 2009 in Prozent}

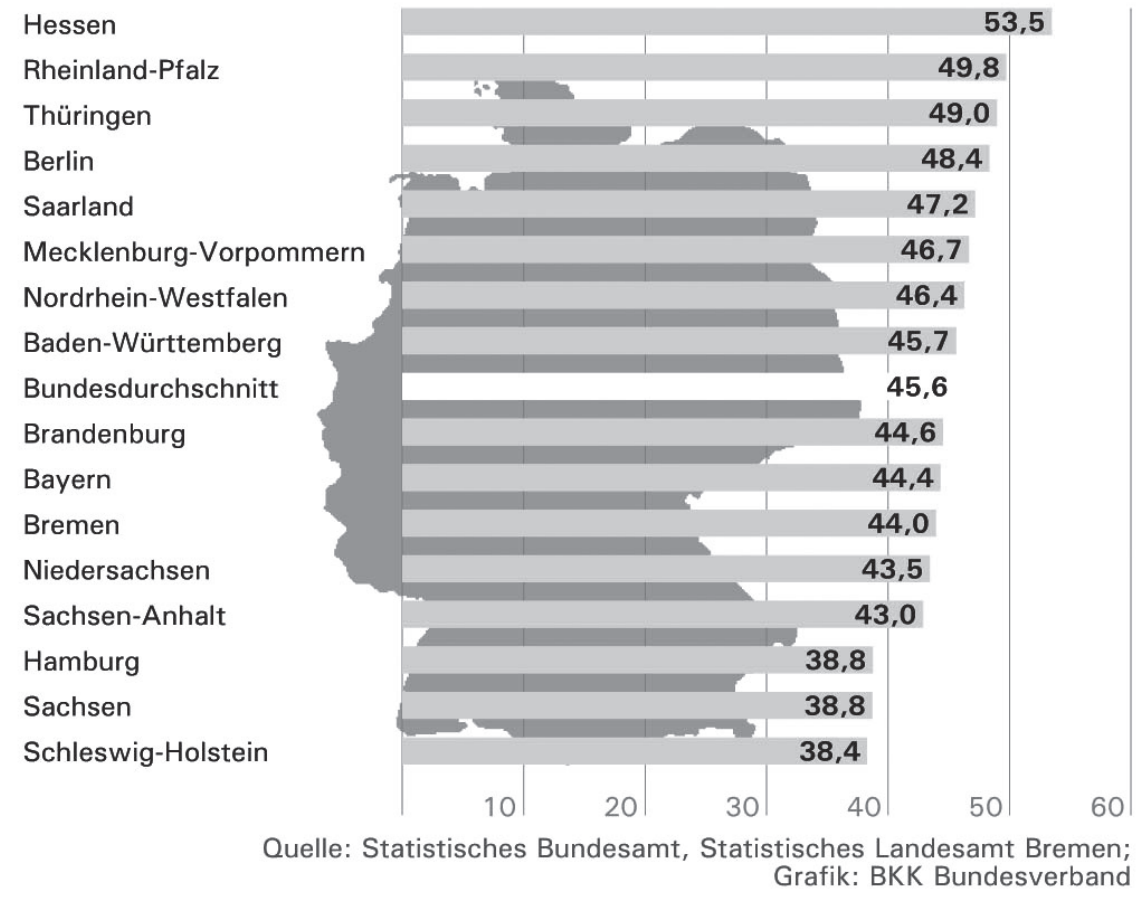

\section{Pflegeheimdichte nach Bundesländern 2009 Anzahl der Pflegeheime je 100.000 Einwohner}

Schleswig-Holstein
Niedersachsen
Sachsen-Anhalt
Mecklenburg-Vorpommern
Sachsen
Thüringen
Brandenburg
Bremen
Bundesdurchschnitt
Baden-Württemberg
Saarland
Bayern
Nordrhein-Westfalen
Hessen
Rheinland-Pfalz
Berlin
Hamburg

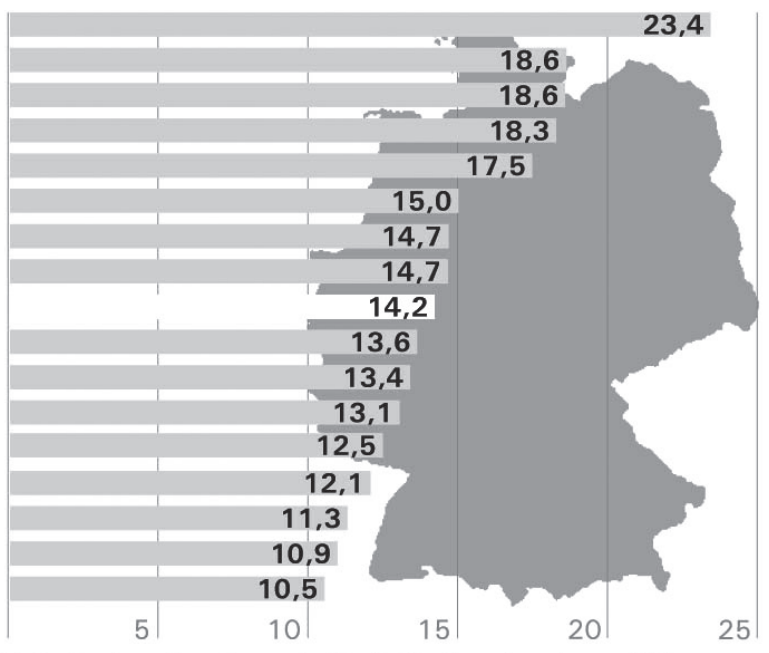

Quelle: Statistisches Bundesamt, Statistisches Landesamt Bremen; Grafik: BKK Bundesverband

folgt von dem Landkreis Osterode am Harz (Niedersachsen) und der badenwürttembergischen Stadt Baden-Baden mit 47,4 \% bzw. 46,9\%. Die kreisfreie Stadt Straubing in Bayern wies einen Anteil von 45,2\% auf. Knapp davor ist der bayerische Landkreis Rosenheim mit einem Anteil von 45,4\% platziert. Der geringste Anteil vollstationärer Dauer- pflege wurde im Emsland (Niedersachsen) mit 14,0\% festgestellt. Geringe Anteile hatten auch das thüringische Sömmerda (15,2 \%) und der bayerische Landkreis Aschaffenburg (16,0\%). « ${ }^{37}$

Ein letztes Schlaglicht auf regionale Unterschiede soll mit den »Nettoausgaben für die Hilfe zur Pflege je Einwohner" geworfen werden. Hier wurden
2008 in den Stadtstaaten die höchsten Nettoausgaben verzeichnet. In Berlin und Hamburg waren es 83 Euro pro Einwohner, gefolgt von Bremen mit 62 Euro je Einwohner. Besonders auffällig ist »der Unterschied zwischen den alten und den neuen Bundesländern: Während in Westdeutschland (ohne Berlin) durchschnittlich 35 Euro je Einwohner an Hilfe zur Pflege gewährt wurde, waren es in Ostdeutschland (ohne Berlin) nur 13 Euro. Bundesweit lag der Durchschnitt bei knapp 34 Euro je Einwohner. $\ll^{38}$ Dass Pflegebedürftigkeit mit Sozialhilfe-Bedarf verbunden ist, ist ganz offensichtlich multifaktoriell bedingt und lässt sich auch nicht so einfach mit west-östlichen Stereotypen zur Einkommens- und Sozialstruktur erklären.

\section{Regionale Unterschiede: Fazit}

Die Thematisierung regionaler Unterschiede hat im Moment in der Gesundheitspolitik Hochkonjunktur. Die »kleinräumige« Diskussion über Versorgung und Bedarfsplanung etc. hat die Pflegeversicherung bisher jedoch noch nicht erreicht. Wenn man aber Pflegepolitik sinnvoll anpacken will, wäre die regionale Dimension sicher in Zukunft stärker zu beachten. Dabei werfen alle angeführten Daten erst einmal mehr Fragen auf, als sie Antworten geben. Das ist auch das, was wenigstens alle seriösen Wissenschaftler in der gesundheitspolitischen Debatte um die Regionalitätsaspekte betonen. Trotzdem könnte gerade die Analyse regionaler Verhältnisse in der Pflege in Zukunft zu einem Lernprogramm für erfolgreiche Praxis, d.h. für Pflegequalität und eine effizientere Organisation der Pflege werden.

\section{Die Analyse regionaler Verhältnisse in der Pflege könnte zu einem Lernprogramm für erfolgreiche Praxis werden.}

37 Statistisches Bundesamt: »Pflegestatistik 2007 - Kreisvergleich «, erschienen im Februar 2010, Seite 10

38 Statistisches Bundesamt, Hilfe zur Pflege, 2008, Seite 17 